units. "The geometric math is in your favor on this one," says Michael Joyner, professor of anesthesiology at the Mayo Clinic and national clinical lead on the program. "There is an amplification effect." About $70 \%$ of the general public are considered eligible to donate blood. Applying the same rule of thumb to the present crisis suggests that $70 \%$ of those who recover may be suitable donors, but that represents an upper limit at best.

The FDA's recently published guidance recommends a minimum neutralizing antibody titer of 1:160 (meaning that a 1-in-160 dilution of a given unit of plasma has activity against the virus); the European Commission's recent guidance recommends a 1:320 titer. Antibody titers are generally tested with high throughput enzyme-linked immunosorbent assay (ELISA), but most sites do not have ELISA-based SARS-CoV-2 testing in place as yet. "Right now, we're just working under the assumption that it works," says Joyner. In time, the study will analyze the relationship between clinical outcomes and neutralizing antibody titers present in the donated plasma.

If convalescent plasma can be considered the first leg in a therapeutic relay race, $\mathrm{H}-\mathrm{Ig}$ is the second. Whereas convalescent plasma requires minimal manipulation screening for transmissible pathogens and pathogen inactivation are the main processes employed - $\mathrm{H}$-Ig is a standardized pharmaceutical product, made from the purified antibody fraction. As such, it requires full clinical development before approval. "One way of thinking about this is the convalescent plasma is the starting material," says Julie Kim, president of Takeda's business unit for plasma-derived therapies. But the end product differs from convalescent plasma in its purity and composition: $\mathrm{H}$-Ig principally contains the immunoglobulin $\mathrm{G}$ antibody fraction of the donated plasma, whereas convalescent plasma contains all the blood fluid minus the cellular fraction. $\mathrm{H}$-Ig is, therefore, more concentrated and more potent than convalescent plasma. "It's consistent, it's scalable, it's reliable; it can be shipped around the world," says Kim. Tokyo-based Takeda and CSL Behring have established the CoVIg19 Plasma Alliance to accelerate the development of a single, unbranded H-Ig product for COVID-19. They have been joined by four other blood-product firms: Biotest, based in Dreieich, Germany; Bio Products Laboratory, of Elstree, UK; Les Ulis, France-based LFB; and Lachen, Switzerland-based Octapharma. Microsoft is also supporting the initiative and has developed the CoVIg-19 Bot, a self-screening tool for potential donors to assess their eligibility and to locate the nearest plasma collection center.

Other firms are developing additional products, including Barcelona-based Grifols, which will produce a H-Ig product at its facility in Clayton, North Carolina, and Emergent BioSolutions, which is developing both a human $\mathrm{H}-\mathrm{Ig}$ product, COVID-HIG, and a product based on equine antibodies, developed from the plasma of horses immunized with SARS-CoV-2.

There is, of course, no clean 'baton exchange' - to extend the relay metaphor between convalescent plasma and $\mathrm{H}$-Ig; their use will inevitably overlap, even if they become available at different timescales. "We absolutely believe there is room for both. Convalescent plasma has more immediacy. Ours will take a while to produce," says Bill Mezzanotte, head of research and development at CSL Behring. Before the formation of the alliance, Takeda had aimed to have $\mathrm{H}$-Ig available in 9-18 months, Kim says. "With the alliance, we hope to beat that."

Efforts to develop recombinant $\mathrm{H}$-Ig-like products are underway as well. Most advanced is SAb Biotherapeutics, which is collaborating with CSL Behring and BARDA to develop SAB-185, a polyclonal human antibody cocktail obtained from the plasma of transgenic cattle immunized with the SARS-CoV-2 spike protein. The platform, which involves introducing the full complement of human antibody genes on an artificial chromosome, has spent two decades in development. "We 'bovinized' portions of the human antibody genes," SAB CEO and cofounder Eddie Sullivan says. The amino acid sequence of the resulting antibodies are still fully human, but the genes contain bovine regulatory elements that optimize their expression in bovine plasma cells. The company is following a hybrid regulatory pathway originally opened more than a decade ago by Revo Biologics (formerly GTC Biotherapeutics) for the production of Atryn (recombinant human antithrombin) in the milk of transgenic goats. It involves the FDA's Center for Veterinary Medicine (CVM) and its Center for Biologics Evaluation and Research. "CVM reviews the animal platform; CBER regulates the antibody product itself," Sullivan says.

SAb has already demonstrated the feasibility of the approach with a different antibody cocktail, SAB-301, directed against the spike protein of another lethal coronavirus, Middle Eastern respiratory syndrome coronavirus (MERS-CoV).

\section{Genomic search for COVID-19 severity clues}

Several large-scale genomic studies are gearing up to pin down the genes underlying individual responses to COVID-19. Genomics England is partnering with Illumina and the UK National Health Service (NHS) to sequence the genomes of 35,000 patients: 15,000 with mild symptoms and 20,000 who required intensive care. A separate group, UK BioBank, is assembling research materials from data on patients with COVID-19 collected by Public Health England, which they will share with 15,000 approved researchers from 85 countries. Finally, nearly 1,000 human geneticists formed the COVID-19 Host Genetics Initiative, organized by the Institute for Molecular Medicine Finland.

Private companies are also contributing. Consumer genomics companies 23andme and Ancestry.com are setting up programs to link COVID-19 symptoms and experiences with genomic information contained in their databases. In the first few weeks 23 andme identified 6,000 people who tested positive for SARS-CoV-2, and in May stepped up its efforts to assemble thousands more by offering free genome analysis to non-members who qualify and are willing to join a COVID-19 research program. Ancestry. com has asked its customer base to fill out a survey on their COVID-19 experiences, the results of which they will share with qualified researchers at no cost.

In the largest study of any kind on COVID-19, a team led by Ben Goldacre at the University of Oxford reported in the preprint server medRxiv that Asians and members of minority groups in the UK face an increased risk of death by COVID-19. Analyzing health records of over 17 million patients from the NHS, the researchers found that comorbidities (such as heart disease, diabetes, asthma and obesity), largely assumed to be behind the increased risk, accounted for only about half the disparity in deaths between minority and white populations. A smaller study by the Oxford Royal College of General Practitioners looking at risk factors for testing positive for COVID-19 was published in mid-May.

Published online: 9 June 2020 https://doi.org/10.1038/s41587-020-0567-3 